\title{
A breaker-zone wave energy converter
}

\author{
M. Negri ${ }^{1}$, F. Clerici ${ }^{1}$ and S.Malavasi ${ }^{1}$ \\ ${ }^{1}$ D.I.I.A.R. \\ Politecnico Di Milano \\ Campus Leonardo - Piazza Leonardo da Vinci 32, 20133 Milano (Italy) \\ Phone: +39 2399 6261, e-mail: marco.negri@polimi.it, francesco.clerici@mail.polimi.it, stefano.malavasi@polimi.it
}

\begin{abstract}
In this work we present a preliminary study on a laboratory model of a breaker zone wave energy converter (WEC). It is a heave-surge point absorber, composed by two distinct elements, a buoy and a paddle, which allows the conversion of the wave energy in near-shore region, just before waves break.

The model was tested in an experimental wave flume, on a mild slope beach, with different waves breaking on the beach.

We found efficiency ranging from 20 to $40 \%$ with respect to the power of the oncoming wave.
\end{abstract}

\section{Key words}

Wave energy, point absorber, nearshore, breaker-zone, heave, surge.

\section{Introduction}

Nowadays, various type of wave energy converters have been designed for harvesting wave energy in shoreline, nearshore and offshore sites, and continuous efforts are made to find a technology that is economically applicable and energetically efficient. Shoreline WEC are generally overtopping devices, terminators or oscillating water columns, while near-shore and offshore WEC are generally oscillating bodies (see [1] for a comprehensive review).

In the history of wave energy harnessing, offshore and shoreline sites have been preferred for wave energy harvesting. Among all sea sites, offshore ones (depth $>40-$ $50 \mathrm{~m}$ ) have the greatest availability of wave energy, but have also high costs of installation, maintenance and connection of the WEC to electrical grid, growing with water depth and distance from the shoreline [1]. On the other hand, shoreline sites have the aforementioned advantages of a convenient position, but they are characterized by much less energy availability, due to the wave energy dissipation which occurs in shallow water; however, wave refraction and diffraction can concentrate energy along the coasts of certain places, like promontories or isles (hot spots). In the last years, nearshore sites are being revalued for wave energy extraction, since it has been found that the real exploitable wave energy is not much less than in the offshore locations, at least up to a water depth of $8 \mathrm{~m}$ [2]. Indeed, a relevant part of the offshore gross energy is not exploitable by any WEC, because it comes with sea storms during small periods of time; the nearshore region acts as a natural filter towards these highly energetic seas [2].

The breaker zone is the portion of the near-shore where wave begin breaking, with typical depth of 5-10 m (but even less, depending on the beach morphology and the wave spectrum of the site). In this zone, wave energy is dissipated mostly through bottom friction and finally through wave breaking; the energy loss due to bottom friction will be highly dependent on the beach slope: mild-steep beach will cause a lower energy loss than a gently slope beach, because of the minor distance that wave have to travel in shallow water [2,3]. In sea sites with beach with mild-steep slopes, this means that wave, before breaking, can still contain a great part of the energy that had offshore; for this reason, the breaker zone could be the last advantageous position for harnessing waves before they dissipate the great part of their energy.

To the authors knowledge, the only WECs that work with breaking waves are oscillating water columns [4], while there are not point absorbers specifically designed for harvest these waves, neither in an industrial prototype nor in a research study. Indeed, point absorbers, and more specifically oscillating bodies, are developed for work with non-breaking waves, while in the breaker zone waves are highly non-linear and are characterized by a high mass transport.

The device we have studied in this work, named EDS (Energy Double System), is a point absorber which aims to harness energy in the breaker zone. EDS is a patent of Tecnomac ${ }^{\circledR}$ (www.tecnomac.it). The idea behind EDS is to exploit heave and surge wave force with a single machine, by means of a float and a paddle mounted on the same arm and reacting with each other, positively if it possible. Float and paddle are aligned along the wave direction propagation (Fig. 1), and paddle is mounted behind the float. The float of EDS is a heaving buoy (it is similar to a module of the Danish Wavestar ${ }^{\circledR}$ prototype, http://wavestarenergy.com/). The paddle of EDS collects the surge wave force; it is not an oscillating 
body, as it works only in the direction of wave propagation. The paddle has low inertia, an immersion comparable to the wave height, and it works well with waves about breaking, because they have a strong mass transport; as a matter of fact, a similar paddle tested on non-breaking waves by Hazlett et al. [5] gave quite low efficiencies.

The EDS challenge is to join these two devices, that, singularly taken, work at best with two different kind of waves, and hence at different locations: an heaving buoy, which is generally designed for offshore sites [6], and a paddle, which significantly works only if the waves are close to break. Therefore the EDS system is suitable for the breaker-zone, at depths of 3-10 m, depending on site characteristics and wave spectrum. Although a heaving buoy may not work at best in this zone, the presence of the paddle compensates this deficiency. The goal of EDS is to keep a relatively high efficiency with the advantages that come from positioning a system near the coast.

As each wave breaks at a different depth, positioning of EDS is crucial: it must intercept a large part of the waves before they break and lose energy. On the other side, small waves will meet the paddle when they are still nonbreaking, and so they will transfer a little energy to the paddle; in principle, this will be compensated by an increase of float efficiency, due to more "linearity" of these waves, and due to the fact that the paddle does not affects the float movement. We can see this as a system versatility: once EDS is optimized on a reference wave, bigger waves will be harvested mostly by the paddle, while smaller waves by the float.

\section{Experimental setup}

The experimental campaign was carried out in the Hydraulics Laboratory of Politecnico di Milano. A wave flume, $30 \mathrm{~m}$ long, $1 \mathrm{~m}$ wide, $0.7 \mathrm{~m}$ high, equipped with a piston wavemaker, was utilized for the experimental tests. A smooth beach with a slope of $7^{\circ}$ was placed at the end of the channel. The water depth in the channel was the same for all the tests, $0.4 \mathrm{~m}$. Three different waves were used in the tests, characterized by increasing power (Table 1). The first two waves have similar ripidity, while wave $n^{\circ} 3$ is a shorter wave, characterized by a high ripidity.

Table 1: Characteristics of the experimental waves, evaluated far from the beach.

\begin{tabular}{|c|c|c|c|c|}
\hline Wave & $\mathrm{H}[\mathrm{mm}]$ & $\mathrm{T}[\mathrm{s}]$ & $\mathrm{L}[\mathrm{m}]$ & $\mathrm{P}[\mathrm{W} / \mathrm{m}]$ \\
\hline 1 & 47 & 1.02 & 1.49 & 2.51 \\
\hline 2 & 65 & 1.2 & 1.83 & 5.73 \\
\hline 3 & 88 & 1.02 & 1.51 & 9.09 \\
\hline
\end{tabular}

Waves measurements were taken by means of two capacitive wave gauges, far from the beach and the wavemaker, without the model in the channel. Wave power per unit length, $P$, was calculated as:

$P=\frac{1}{8} \rho g H^{2} c_{g}$ where $\rho, g, H$ are the water density, the gravity and the wave height respectively, while $c_{g}$ was calculated as:

$c_{g}=\frac{c}{2}\left(1+\frac{2 k h}{\sinh (2 k h)}\right)$

where $c$ is the wave celerity, $h$ the water depth, $k=2 \pi / L$ the wave number, with $L$ being the wave length. We evaluated wave length by correlation of the signals of the wave gauges. On the beach the waves shortened and their height increased; we considered null the energy dissipation along the beach, as its length was 1-2 times the wave length.

The scheme of EDS model reported in Fig. 1 is composed by a main arm, hanged over the water surface, which supports the float on one side, and the damping system on the other side, which has the task of dissipating the energy captured by the float. The float is composed by an hemispherical part in the bottom and a cylindrical part over it. A second arm, which holds the paddle at its lower end, is hinged on the main arm, between the fixed hinge and the float. The paddle has cylindrical shape. The superior end of the paddle arm is connected to a fixed point of the main arm through a spring.

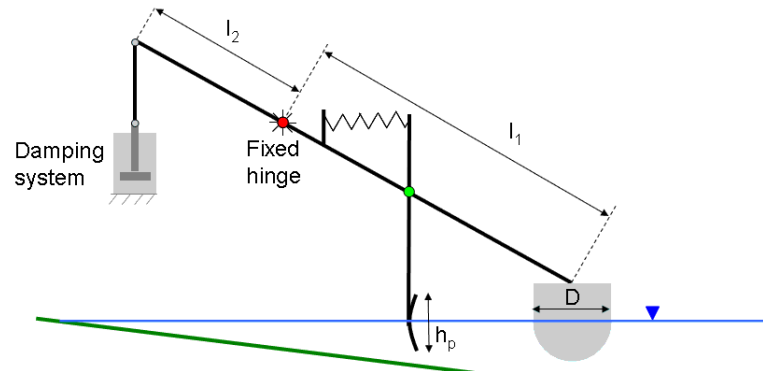

Fig. 1: EDS model's scheme

The damping system is constituted by a porous disc immersed in cylindrical case filled with water (Fig. 2). The porous disc can move vertically in the cylindrical case. The cylinder-disc interface is not watertight: the disc has a diameter of $105 \mathrm{~mm}$, the cylinder has a diameter of $112 \mathrm{~mm}$. The disc is supported by a stem, which is vertically sliding in a low friction bearing, and which is connected to the main arm through leverages. A load cell and a distance sensor was mounted on the disc stem, in order to measure forces and movements, and so calculate the dissipated energy.

The paddle of the model was not equipped with a dissipating system, but with spring, which is a conservative one. We made the assumption that the energy harvested by the paddle could be calculated as the spring potential energy accumulated when the wave pushes the paddle. 


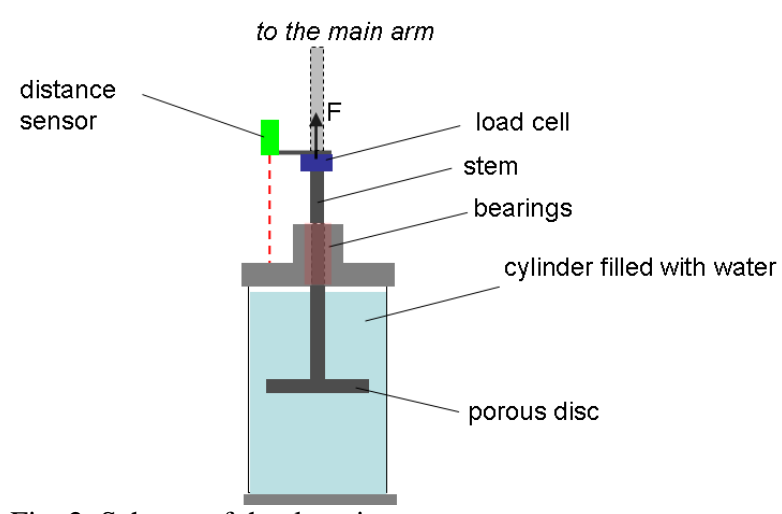

Fig. 2: Scheme of the damping system

This assumption arises from the type of movement of the paddle and from the need to simplify the system, at least in this first phase of study. Under the excitation of the waves, the paddle movement is not harmonic and it is unidirectional (in the direction of wave propagation); moreover, paddle inertia is low compared to the spring force. A distance sensor was mounted on the spring to measure its elongation and so calculate force and energy, knowing the spring stiffness.

The total efficiency of the system (capture width ratio, $C W R$ ) was calculated as:

$$
C W R=\frac{P_{e x}}{P \cdot D}
$$

Where $P_{e x}$ is total power extracted by the EDS system, $P$ is the wave power per unit length, and $D$ is the diameter of the float (and the paddle width). In this model, the paddle width was equal to the float diameter; we thought that this was the basic condition to start with for the testing of the EDS concept. Further studies are needed to determine the effect of a wider or different shaped paddle. In Fig.3 a photo of the EDS model is reported.

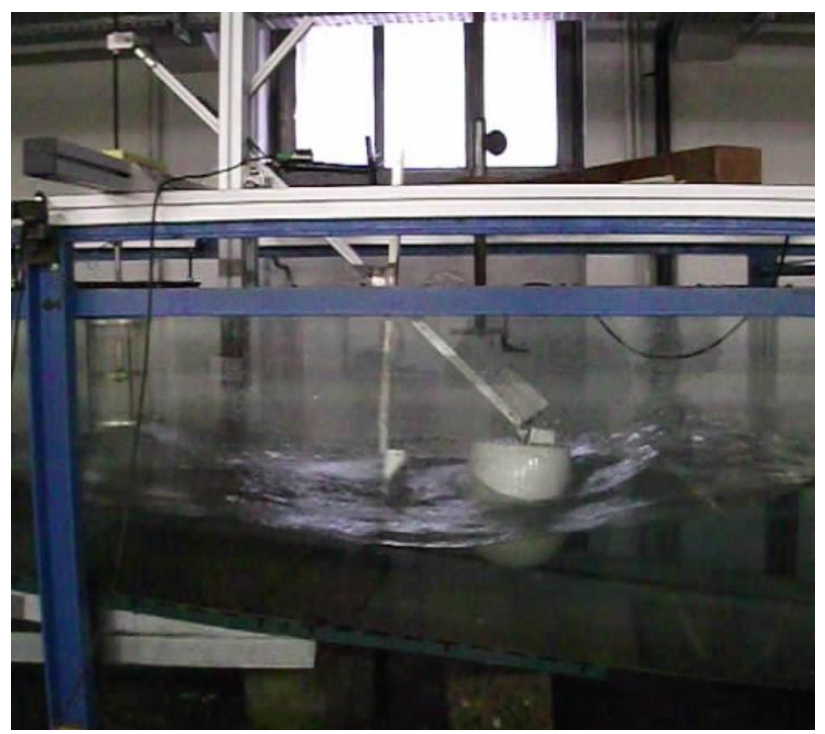

Fig.3: EDS model during the experimental campaign

The model had many parameters that could be varied (geometrical and dynamical). In this first experimental campaign, we decided to fix some of them (reported in
Table 2, in future tests they also will be varied), and optimize the model varying the remaining parameters.

Table 2: Model dimensions not varied during tests

\begin{tabular}{|l|l|}
\hline Float diameter $D[\mathrm{~m}]$ & 0.20 \\
\hline Float total height $[\mathrm{m}]$ & 0.21 \\
\hline Paddle width $[\mathrm{m}]$ & 0.20 \\
\hline Paddle height $h_{p}[\mathrm{~m}]$ & 0.12 \\
\hline Float arm $l l[\mathrm{~m}]$ & 0.67 \\
\hline Damper arm $l 2[\mathrm{~m}]$ & 0.41 \\
\hline
\end{tabular}

In all tests the spring and the damper were the same. The spring stiffness was $\mathrm{k}=196 \mathrm{~N} / \mathrm{m}$. Damping generated by the disc in water was almost quadratic, and its value changed depending on the motion amplitude of the disc. In the tests the average damping was $20-30 \mathrm{~N} / \mathrm{m} \cdot \mathrm{s}$.

Dealing with monochromatic waves, all acquired data was phase-averaged. Mean power over a cycle captured by the paddle was calculated as:

$$
P_{\text {paddle }}=\frac{1 / 2 K x^{2}}{T} \text { (4) }
$$

where $\mathrm{x}$ is the maximum elongation experienced by the spring during the average cycle of work, $K$ is the spring stiffness and $T$ is the average period of oscillation.

Mean power over a cycle captured by the float was calculated as:

$P_{\text {float }}=\frac{\int_{T} F_{D} \cdot v \cdot d t}{T}$

where $F_{D}$ is the damping force on the disc and $v$ is the velocity of the disc.

Total extracted power $P_{e x}$ was calculated as:

$P_{\text {ex }}=P_{\text {float }}+P_{\text {paddle }}$

\section{Results}

EDS is quite a complex machine, because it is composed by two different devices that interact between each other; as before mentioned, there are a lot of parameters that can be varied to find an optimal configuration, at least in the first stage of research on the EDS. This also could be seen as an advantage, because it may be possible to adapt EDS to different sea sites. In this work, we analyzed the effect of some geometrical and dynamical parameters of EDS on its efficiency, reaching to a first interesting configuration of the model.

The optimization was made maximizing the efficiency of the system for the three wave considered, giving more importance to the efficiency of the system when 
subjected to wave $n^{\circ} 1$, because wave $n^{\circ} 1$ gave the highest efficiencies among the three wave tested.

The parameters investigated in this work were: system mass, water depth, distance between paddle and float, paddle immersion. Using the configuration reported in Table 2, we started to investigate the efficiency of model varying its position with respect the shoreline, therefore the water depth $h$, and its mass, placing supplementary mass $m_{\text {sup }}$ on the float (Fig.3). During this first series of tests we maintained constant the paddle immersion in still water at $0.06 \mathrm{~m}$ and the paddle-buoy distance at $0.28 \mathrm{~m}$. The maximum value of $m_{\text {sup }}$ was the one with which the float did not hit the floor during its oscillation or did not bring to the total immersion of the float.

The mass of the parts of the model, with their arm with respect to the fixed hinge, is reported in Table 3.

Table 3: Mass of the model

\begin{tabular}{|l|c|c|}
\hline Part & Mass $[\mathbf{K g}]$ & $\begin{array}{c}\text { Distance from the } \\
\text { fixed hinge [m] }\end{array}$ \\
\hline Float & 0.44 & 0.67 \\
\hline Supplementary mass $m_{\text {sup }}$ & $0-1-2-3$ & 0.67 \\
\hline Main arm & 0.71 & - \\
\hline Paddle system & 1 & 0.4 \\
\hline Disc system & 0.8 & 0.41 \\
\hline
\end{tabular}

Fig. 4a reports the $C W R$ for different supplementary mass on the system and for the lower distance from the shoreline, which correspond to the lower water depth $h=0.15 \mathrm{~m}$. In this case, we can observe that increasing the supplementary mass, $C W R$ significantly increases, especially for wave 2 . In this configuration it was not possible to increase the mass over $2 \mathrm{~kg}$ because the float hit the floor.

Fig. 4b and Fig. 4c depict what happens increasing the distance of the system from the shoreline therefore increasing the water depth. It is interesting to note that for $h=0.20 \mathrm{~m}$ the efficiency of the system increases with the increase of the supplementary mass. Increasing the water depth to $h=0.25 \mathrm{~m}$, we observe that the efficiency of the system is significantly influenced by the type of wave.

To better understanding of the system behaviour we decomposed the measured $C W R$ in three contributions: efficiencies of the paddle, of the float during the downward movement (F-down) and of the float during the upward movement (F-up). Fig. 5 reports the decomposed $C W R$ contributions for wave 1 as a function of the supplementary mass $m_{\text {sup }}$ and the water depth $h$. As expected, float efficiency increases with increasing depth, while paddle efficiency increases as depth decreases. We can also see that the upward phase of the float is always more energetic than the downward one.

We have obtained the highest value of $C W R$ with $\mathrm{h}=0.15$, $m_{\text {sup }}=2 \mathrm{~kg}$, wave $\mathrm{n}^{\circ} 1$; notwithstanding we choose the configuration $\mathrm{h}=0.2 \mathrm{~m}, m_{\text {sup }}=3 \mathrm{~kg}$, because it ensured a fairly good $C W R$ for all the three waves.

With the latter configuration we proceeded with tests varying the distance between paddle and float and the paddle immersion. The maximum distance between paddle and float depended by the physical constraints of the model.

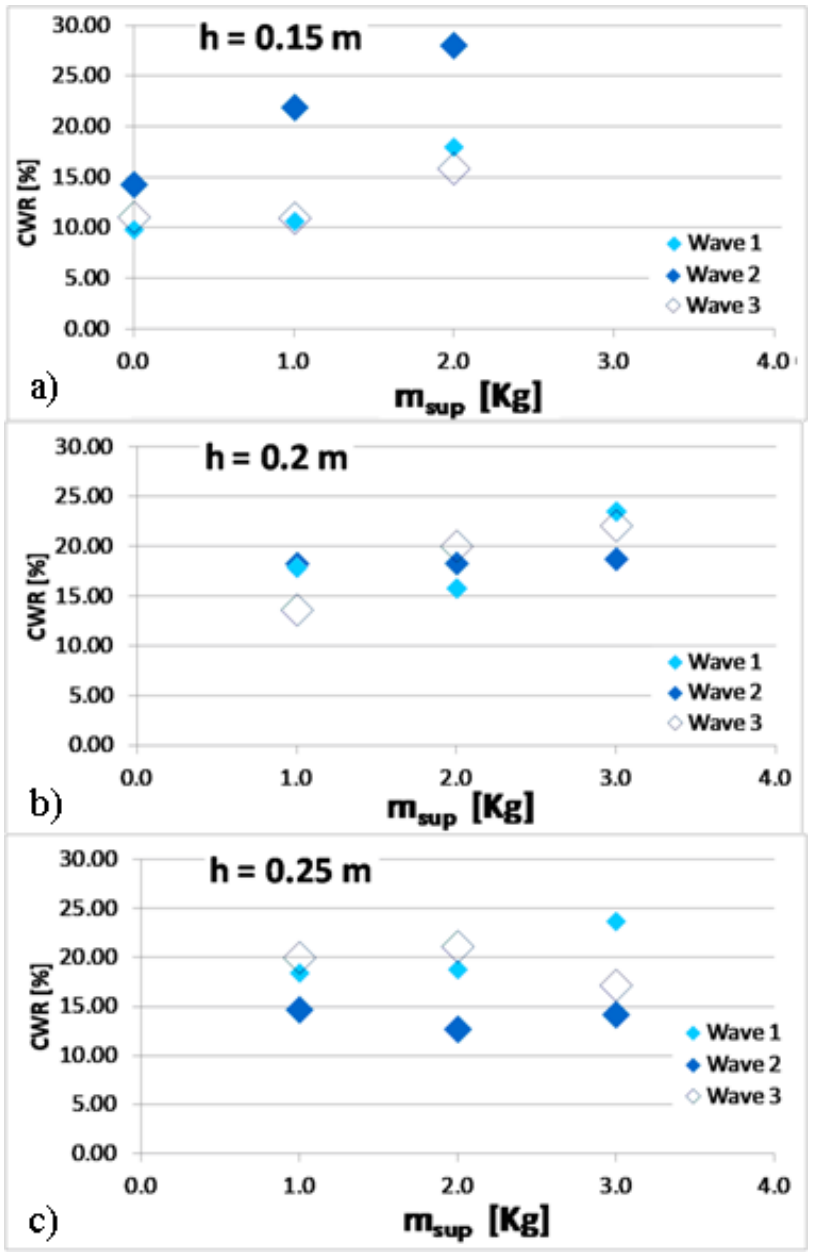

Fig. 4: $C W R$ vs. supplementary mass on the system $m_{\text {sup }}$ for difference water depths: a) $h=0.15$; b) $h=0.20 ; h=0.25$.

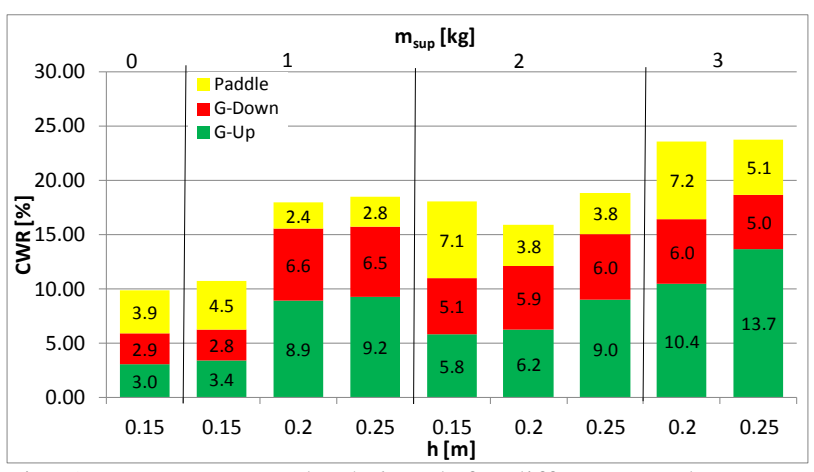

Fig. 5: $C W R$ vs. water depth $h$ and for different supplementary mass $m_{\text {sup }}$ for wave 1 .

In Fig. $6 C W R$ as a function of paddle immersion in still water $(d)$, for different values of paddle-float distance $\left(D_{\text {paddle-float }}\right)$ is reported. With $D_{\text {paddle-float }}=0.04 \mathrm{~m}$ (Fig. 6a), we can see that $C W R$ on average decreases with paddle immersion. With $D_{\text {paddle-float }}=0.10 \mathrm{~m}$ (Fig. 6b) $C W R$ on average increases with paddle immersion. The highest $C W R$ are reached for $D_{\text {paddle-float }}=0.16 \mathrm{~m}$ (Fig. 6c) and an immersion of $d=0.09 \mathrm{~m}$. In this configuration, the paddle is never surmounted by the wave crest. In the experimental range considered the CWR puts in evidence that the paddle immersion should be at least one wave height of the incident wave. We do not exclude that a 
paddle which extends deeper could harness more energy. It is reasonable to assume that the optimal distance between float and paddle is linked to the wave length (at the float and paddle location), because of the mutual influence of the float and the paddle: as the float moves, it raises and lower the paddle in the water, and in turn, the paddle, when it receives the wave push, immerses the float. To obtain a high efficiency, this mutual influence must be performed so that paddle and float are in the correct position with respect to the oncoming wave. The measures of wave length on the beach shows that optimal distance $D_{\text {paddle-float }}$ is $1 / 9-1 / 6$ of the wave length.

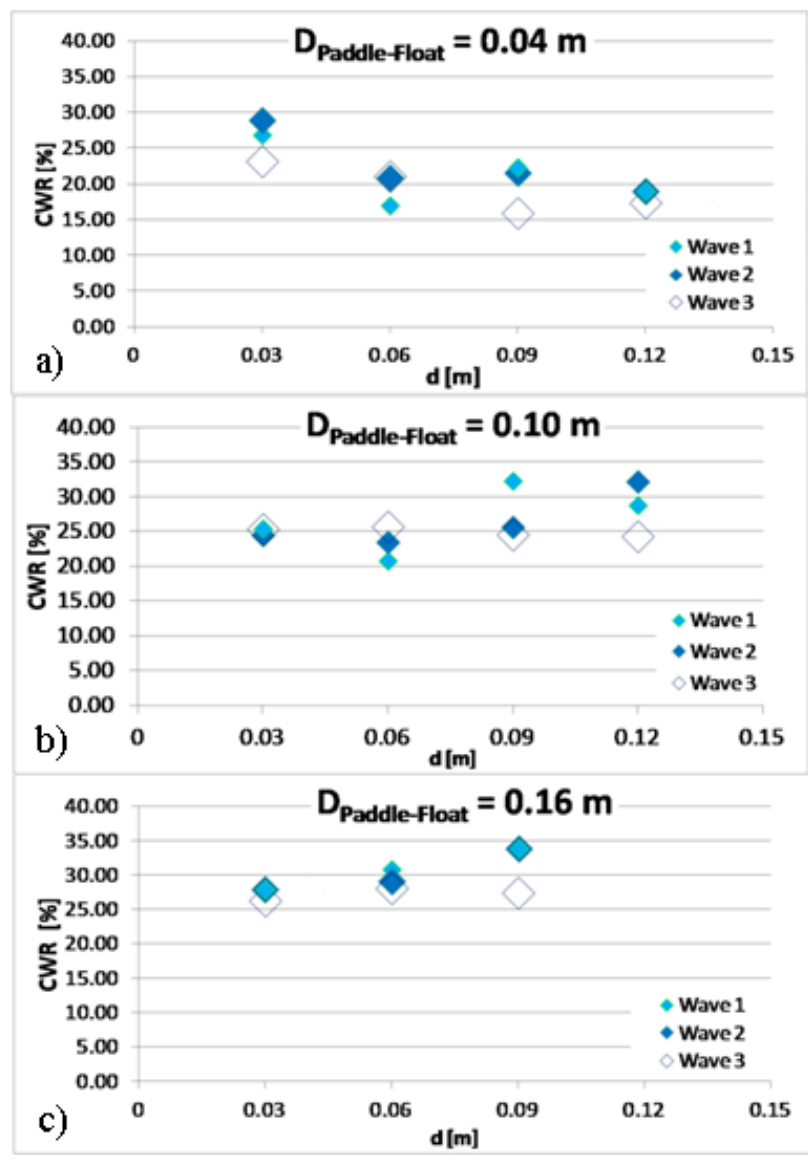

Fig. 6: $C W R$ vs. paddle immersion $d$ for different distances between paddle and float waves and. a) $D_{\text {paddle-float }}=0.04$; b) $D_{\text {paddle-float }}=0.10 ; D_{\text {paddle-float }}=0.16$.

As done before, we show the partial CWRs of the system for wave 1 (Fig. 7). We can see that paddle $C W R$ has a maximum for $\mathrm{d}=0.09 \mathrm{~m}$ for all the cases, and upward phase of float is almost always more energetic than downward phase, as already noted earlier.

We have also investigated other parameters of EDS model, that is the inclination of the main arm and the inclination of the spring connected to the paddle arm. We noted that a horizontal spring and a quite inclined main arm make the system more efficient, and that inclination of the main arm has an effect on the repartition of the energy extracted by the float during its oscillation: increasing the inclination of the main arm, the power of the upward phase of the float increases at the expense of the power of the downward phase. The optimal configuration found is the one of Fig. 1 (the figure is to scale) and values of parameters are summarized in Table 4. For the system mass, refer to Table 3.

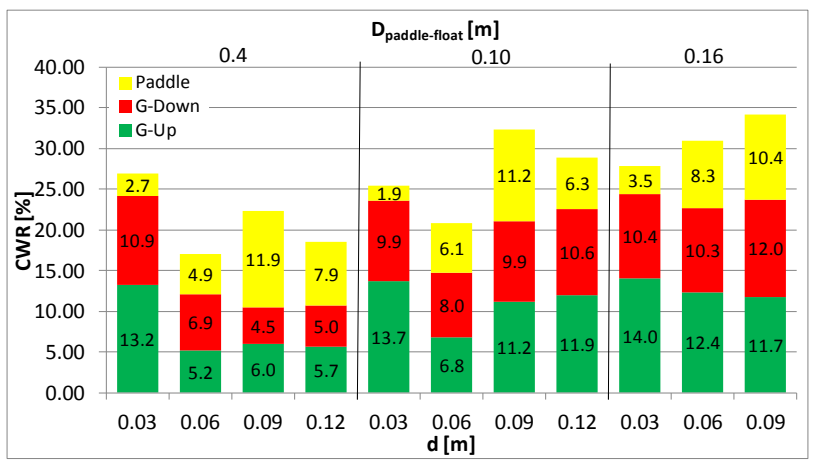

Fig. 7: $C W R$ vs. paddle immersion $d$ and paddle-float distance $D_{\text {paddle-float }}$ for wave 1 .

Table 4: EDS optimal configuration

\begin{tabular}{|l|c|}
\hline Water depth at the float $[\mathrm{m}]$ & 0.2 \\
\hline Paddle immersion in still water $[\mathrm{m}]$ & 0.09 \\
\hline Paddle-buoy distance $[\mathrm{m}]$ & 0.16 \\
\hline Arm inclination $\left[{ }^{\circ}\right]$ & 42 \\
\hline Spring inclination $\left[{ }^{\circ}\right]$ & 0 \\
\hline
\end{tabular}

In Fig. 8, power and efficiency of the system in the best configuration tested are reported for the three waves considered; in this configuration, the upward phase of the float is significantly more energetic than the downward one, and this could motivate the use of a one-way PTO for the float.

We can see that $C W R$ decreases for wave 2 and wave 3, this is due the fact that the system have been mostly optimized for wave 1 , and because there is a wave dimension for which the system works at best, related to the system principal dimension (let us say the float diameter $D$ ). In our case, corresponding to wave 1 we have $H / D=0.24$.

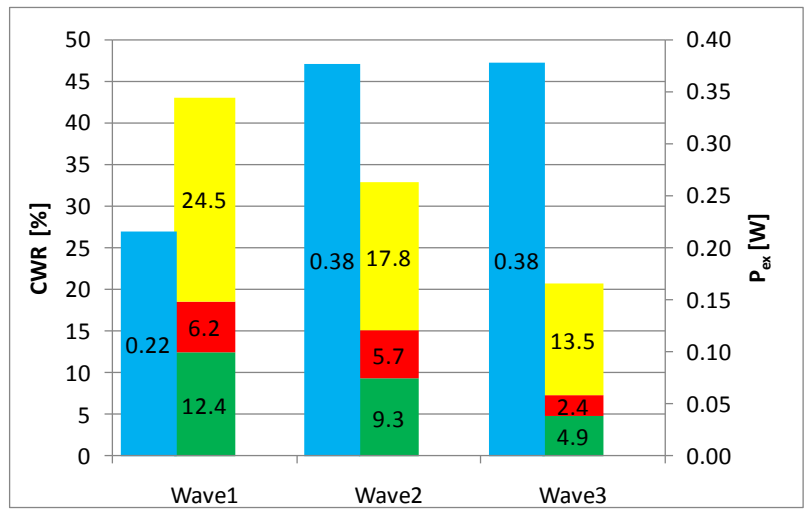

Fig. 8: Efficiency of the system and power extracted.

Generally, the paddle of EDS has an efficiency of 10-25 $\%$, which is quite high if compared with the results of Hazlett et al. [5], who found typical efficiencies less than $10 \%$. For what regards the float, efficiency is lower than the one of Wavestar $\AA$, whose efficiency can reach a 
maximum value of $40 \%$ [7]. This is due to the fact that EDS float works in breaking waves.

An additional test was made to verify the reciprocal effects of float and paddle: the two elements were singularly tested in the configuration and position of the final model arrangement. In Fig. $9 C W R$ of the EDS and of the separated elements (paddle and float) are reported for the three waves considered. For wave 1 we can see that float $C W R$ is not different for the cases of EDS and separated elements, while for wave 2 and wave 3 float $C W R$ is significantly lower in the case of EDS than in the case of separated elements. Conversely, paddle $C W R$ is higher for wave 1 in the case of EDS, while for wave 2 and 3 it is similar in the two cases.

We can see that in all cases the efficiency of EDS is greater than the ones of float and paddle singularly taken. For the smallest wave, efficiency of EDS is even greater than the sum of efficiency of float and paddle singularly taken, and this means that the two elements positively interact: this is an important aspect that should be further pursued.

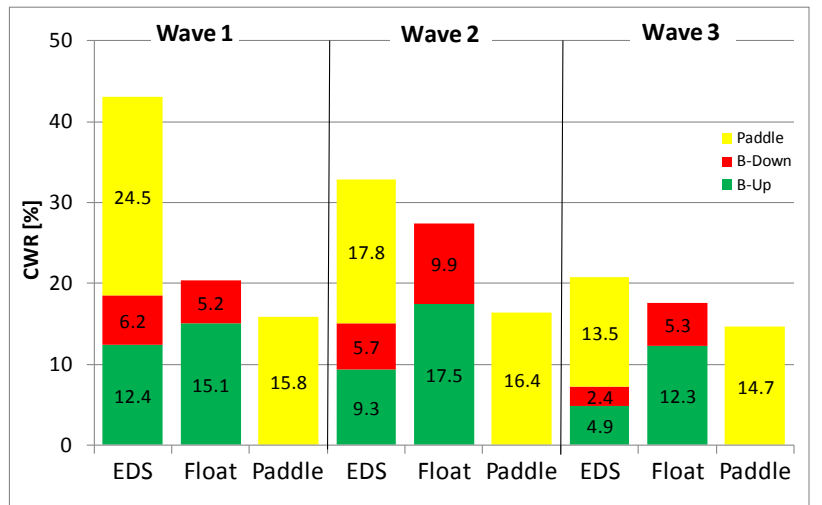

Fig. 9: Efficiency of EDS and of the float and the paddle singularly taken.

\section{Conclusion}

In this work we made a preliminary modelling of the EDS, a wave energy point absorber designed for the breakerzone. We experimentally tested the EDS physical model with monochromatic waves, varying its geometry to find a configuration with best efficiency within the experimental range considered. We found that each parameter of the system, either of the float or of the paddle, influences the motion of the entire system.
The following parameters have a great influence on the system efficiency: water depth, float diameter to wave dimension, system mass, main arm inclination, distance between paddle and float, paddle immersion.

We found that the ideal position of EDS is with the float at water depth of one float diameter, and that immersion of the paddle (in still water) must be at least one wave height. Typical efficiencies (CWR) are between 20-40\%, which are comparable to the ones of Wavestar@ [7].

The extracted energy is divided fairly evenly into the float and the paddle, with the paddle always being more energetic than the float.

Though these results are preliminary and need to be extended, they make us believe that EDS is a promising technology and push to go further in this research, improving the model (making it similar to what a real EDS machine should be) and testing it with a wider range of waves.

\section{Acknowledgement}

This work has been supported by Regione Lombardia and through the "Ricerca industriale e sviluppo sperimentale nel settore dell'efficienza energetica" grant.

We thank Tecnomac for the support in the realization of the experimental set-up.

\section{References}

[1] A. F. de O. Falcao. Wave energy utilization: A review of the technologies. Renewable and Sustainable Energy Reviews, 2009.

[2] M. Folley, T.J.T. Whittaker. Analysis of the nearshore wave energy resource. Renewable Energy 34 (2009) 1709-1715

[3] M.M. Purandare, P.A. Aswatha Narayana. Wave power variation in the near-shore Regions. Coastal Engineering, 11 (1987) 381-390.

[4] E. Martins, FS Ramos, L. Carrilho, P. Justino, L. Gato, L. Trigo, F. Neumann. CeoDouro project: overall design OWC in the new Oporto breakwater. In: Proceeedings of the $6^{\text {th }}$ European Wave Tidal Energy Conference; 2005, p. 273-280

[5] Ben D. Hazlett, Ion I. Inculet, Diana R. Inculet. Electric power generation by "Surfing" water waves. Renewable Energy 34 (2009), 2510-2514.

[6] R. Waters, M. Stalberg, O. Danielsson, O. Svensson, S. Gustafsson, E. Stromsted et al. Experimental results from see trials of an offshore wave energy system. Appl Phys Lett 90(3) [Art No. 034105]

[7] L. Marquis, M. Kramer, P. Frigaard. "Performance Evaluation of the Wavestar Prototype". EWTEC 2011 conference in Southampton, UK. 2011-09-06. 